\title{
Evolutionary Splines for Cepstral Filterbank Optimization in Phoneme Classification
}

\author{
Leandro D. Vignolo, ${ }^{1}$ Hugo L. Rufiner, ${ }^{1}$ Diego H. Milone, ${ }^{1}$ and John C. Goddard ${ }^{2}$ \\ ${ }^{1}$ Research Center for Signals, Systems and Computational Intelligence, Department of Informatics, National University of Litoral, \\ CONICET, Santa Fe, 3000, Argentina \\ ${ }^{2}$ Departamento de Ingeniería Eléctrica, Universidad Autónoma Metropolitana, Unidad Iztapalapa, Mexico D.F., 09340, Mexico
}

Correspondence should be addressed to Leandro D. Vignolo, leandro.vignolo@gmail.com

Received 14 July 2010; Revised 29 October 2010; Accepted 24 December 2010

Academic Editor: Raviraj S. Adve

Copyright ( $) 2011$ Leandro D. Vignolo et al. This is an open access article distributed under the Creative Commons Attribution License, which permits unrestricted use, distribution, and reproduction in any medium, provided the original work is properly cited.

\begin{abstract}
Mel-frequency cepstral coefficients have long been the most widely used type of speech representation. They were introduced to incorporate biologically inspired characteristics into artificial speech recognizers. Recently, the introduction of new alternatives to the classic mel-scaled filterbank has led to improvements in the performance of phoneme recognition in adverse conditions. In this work we propose a new bioinspired approach for the optimization of the filterbanks, in order to find a robust speech representation. Our approach—which relies on evolutionary algorithms—reduces the number of parameters to optimize by using spline functions to shape the filterbanks. The success rates of a phoneme classifier based on hidden Markov models are used as the fitness measure, evaluated over the well-known TIMIT database. The results show that the proposed method is able to find optimized filterbanks for phoneme recognition, which significantly increases the robustness in adverse conditions.
\end{abstract}

\section{Introduction}

Most current speech recognizers rely on the traditional mel-frequency cepstral coefficients (MFCC) [1] for the feature extraction phase. This representation is biologically motivated and introduces the use of a psychoacoustic scale to mimic the frequency response in the human ear.

However, as the entire auditory system is complex and not yet fully understood, the shape of the true optimal filterbank for automatic recognition is not known. Moreover, the recognition performance of automatic systems degrades when speech signals are contaminated with noise. This has motivated the development of alternative speech representations, and many of them consist in modifications to the mel-scaled filterbank, for which the number of filters has been empirically set to different values [2]. For example, Skowronski and Harris [3, 4] proposed a novel scheme for determining filter bandwidth and reported significant recognition improvements compared to those using the MFCC traditional features. Other approaches follow a common strategy which consists in optimizing a speech representation so that phoneme discrimination is maximized for a given corpus. In this sense, the weighting of MFCC according to the signal-to-noise ratio (SNR) in each mel band was proposed in [5]. Similarly, [6] proposed a compression of filterbank energies according to the presence of noise in each mel subband. Other modifications to the classical representation were introduced in recent years [79]. Further, in [10], linear discriminant analysis was studied in order to optimize a filterbank. In a different approach, the use of evolutionary algorithms has been proposed in [11] to evolve speech features. An evolution strategy was also proposed in [12], but in this case for the optimization of a wavelet packet-based representation. In another evolutionary approach, for the task of speaker verification, polynomial functions were used to encode the parameters of the filterbanks, reducing the number of optimization parameters [13]. However, a complex relation between the polynomial coefficients and the filterbank parameters was proposed, and the combination of multiple optimized filterbanks and classifiers requires important changes in a standard ASR system. 
Although these alternative features improve recognition results in controlled experimental conditions, the quest for an optimal speech representation is still incomplete. We continue this search in the present paper using a biologically motivated technique based on evolutionary algorithms (EAs), which have proven to be effective in complex optimization problems [14]. Our approach, called evolutionary splines cepstral coefficients (ESCCs), makes use of an EA to optimize a filterbank, which is used to calculate scaled cepstral coefficients.

This novel approach improves the traditional signal processing technique by the use of an evolutionary optimization method; therefore, the ESCC can also be considered as a bioinspired signal representation. Moreover, one can think about this strategy as related to the evolution of the animal's auditory systems. The center frequencies and bandwidths, of the bands by which a signal is decomposed in the ear, are thought to result from the adaptation of cochlear mechanisms to the animal's auditory environment [15]. From this point of view, the filterbank optimization that we address in this work is inspired by natural evolution. Finally, this novel approach should be seen as a biologically motivated technique that is useful for filterbank design and can be applied in different applications.

In order to reduce the number of parameters, the filterbanks are tuned by smooth functions which are encoded by individuals in the EA population. Nature seems to use "tricks" like this to reduce the number of parameters to be encoded in our genes. It is interesting to note some recent findings that suggest a significant reduction in the estimated number of human genes that encode proteins [16]. Therefore, the idea of using splines in order to codify several optimization parameters with a few genes is also inspired by nature.

A classifier employing a hidden Markov model (HMM) is used to evaluate the individuals, and the fitness is given by the phoneme classification result. The ESCC approach is schematically outlined in Figure 1. The proposed method attempts to find an optimal filterbank, which in turn provides a suitable signal representation that improves on the standard MFCC for phoneme classification.

In a previous work, we proposed a strategy in which different parameters of each filter in the filterbank were optimized, and these parameters were directly coded by the chromosomes [17]. In this way, the size of the chromosomes was proportional to the number of filters and the number of parameters, resulting in a large and complex search space. Although the optimized filterbanks produced some phoneme recognition improvements, the fact that very different filterbanks also gave similar results suggested that the search space should be reduced. That is why our new approach differs from the previous one in that the filter parameters are no longer directly coded by the chromosomes. More precisely, the filterbanks are defined by spline functions whose parameters are optimized by the EA. In this way, with only a few parameters coded by the chromosomes, we can optimize several filterbank characteristics. This means that the search space is significantly reduced whilst still keeping a wide range of potential solutions.

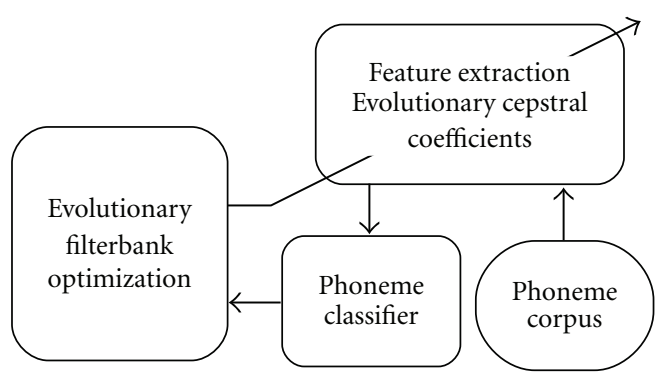

FIGURE 1: General scheme of the proposed method.

This paper is organized as follows. In the following section, some basic concepts about EAs are given and the steps for computing traditional MFCC are explained. Also, a description of the phoneme corpus used for the experiments is provided. Subsequently, the details of the proposed method and its implementation are described. In the last sections, the results of phoneme recognition experiments are provided and discussed. Finally, some general conclusions and proposals for future work are given.

\section{Preliminaries}

2.1. Evolutionary Algorithms. Evolutionary algorithms are metaheuristic optimization methods motivated by the process of natural evolution [18]. A classic EA consists of three kinds of operators: selection, variation, and replacement [19]. Selection mimics the natural advantage of the fittest individuals, giving them more chance to reproduce. The purpose of the variation operators is to combine information from different individuals and also to maintain population diversity, by randomly modifying chromosomes. Whether all the members of the current population are replaced by the offspring is determined by the replacement strategy. The information of a possible solution is coded by the chromosome of an individual in the population, and its fitness is measured by an objective function which is specific to a given problem. Parents, selected from the population, are mated to generate the offspring by means of the variation operators. The population is then replaced and the cycle is repeated until a desired termination criterion is reached. Once the evolution is finished, the best individual in the population is taken as the solution for the problem [20]. Evolutionary algorithms are inherently parallel, and one can benefit from this in a number of ways to increase the computational speed [12].

2.2. Mel-Frequency Cepstral Coefficients. The most popular features for speech recognition are the mel-frequency cepstral coefficients, which provide greater noise robustness in comparison to the linear-prediction-based feature extraction techniques, but even so they are highly affected by environmental noise [21].

Cepstral analysis assumes that the speech signal is produced by a linear system. This means that the magnitude spectrum of a speech signal $Y(f)$ can be formulated as 


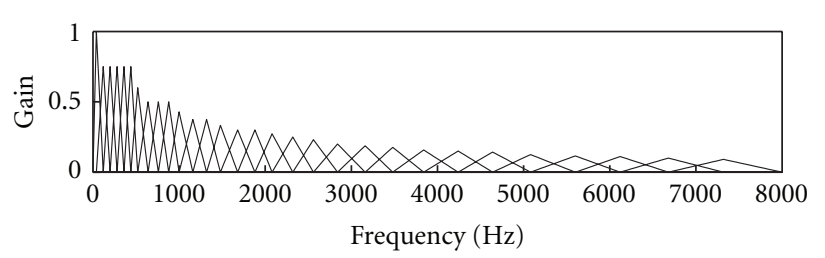

Figure 2: A mel filterbank in which the gain of each filter is scaled by its bandwidth to equalize filter output energies.

the product $Y(f)=X(f) H(f)$ of the excitation spectrum $X(f)$ and the frequency response of the vocal tract $H(f)$. The speech signal spectrum $Y(f)$ can be transformed by computing the logarithm to get an additive combination $C(f)=\log _{e}|X(f)|+\log _{e}|H(f)|$, and the cepstral coefficients $c(n)$ are obtained by taking the inverse Fourier transform (IFT) of $C(f)$.

Due to the fact that $H(f)$ varies more slowly than $X(f)$, in the cepstral domain the information corresponding to the response of the vocal tract is not mixed with the information from the excitation signal and is represented by a few coefficients. This is why the cepstral coefficients are useful for speech recognition, as the information that is useful to distinguish different phonemes is given by the impulse response of the vocal tract.

In order to incorporate findings about the critical bands in the human auditory system into the cepstral features, Davis and Mermelstein [1] proposed decomposing the log magnitude spectrum of the speech signal into bands according to the mel-scaled filterbank. Mel is a perceptual scale of fundamental frequencies judged by listeners to be equal in distance from one another [22], and the mel filterbank (MFB) consists of triangular overlapping windows. If the $M$ filters of a filterbank are given by $H_{m}(f)$, then the log-energy output of each filter $m$ is computed by

$$
S[m]=\ln \left[\int|X(f)|^{2} H_{m}(f) d f\right] .
$$

Then, the mel-frequency cepstrum is obtained by applying the discrete cosine transform to the discrete sequence of filter outputs:

$$
c[n]=\sum_{m=0}^{M-1} S[m] \cos \left(\frac{\pi n(m-1 / 2)}{M}\right), \quad 0 \leq n<M .
$$

These coefficients are the so-called mel-frequency cepstral coefficients (MFCCs) [23].

Figure 2 shows an MFB made up of 23 equal-area filters in the frequency range from 0 to $8 \mathrm{kHz}$. The bandwidth of each filter is determined by the spacing of central frequencies, which is in turn determined by the sampling rate and the number of filters [24]. This means that, given the sampling rate, if the number of filters increases, bandwidths decrease and the number of MFCC increases. For both MFCC and ESCC, every energy coefficient resulting from band integration is scaled, by the inverse of the filter area for MFCC, and by optimized weight parameters in the case of ESCC.

\section{Evolutionary Splines Cepstral Coefficients}

The search for an optimal filterbank could involve the adjustment of several parameters, such as the number of filters, and the shape, amplitude, position, and width of each filter. The optimization of all these parameters together is extremely complex; so in previous work we decided to maintain some of the parameters fixed [17]. However, when considering triangular filters, each of which was defined by three parameters, the results showed that we were dealing with an ill-conditioned problem.

In order to reduce the chromosome size and the search space, here we propose the codification of the filterbanks by means of spline functions. We chose splines because they allow us to easily restrict the starting and end points of the functions' domain, and this was necessary because we wanted all possible filterbanks to cover the frequency range of interest. This restriction benefits the regularity of the candidate filterbanks. We denote the curve defined by a spline by $y=c(x)$, where the variable $x$ takes $n_{f}$ equidistant values in the range $(0,1)$ and these points are mapped to the range $[0,1]$. Here, $n_{f}$ stands for the number of filters in a filterbank; so every value $x[i]$ is assigned to a filter $i$, for $i=1, \ldots, n_{f}$. The frequency positions, determined in this way, set the frequency values where the triangular filters reach their maximum, which will be in the range from $0 \mathrm{~Hz}$ to half the sampling frequency. As can be seen on Figure 3(b), the starting and ending frequencies of each filter are set to the points where its adjacent filters reach their maximum. Therefore, the filter overlapping is restricted. Here we propose the optimization of two splines: the first one to arrange the frequency positions of a fixed number of filters and the second one to set the filters amplitude.

Splines for Optimizing the Frequency Position of the Filters. In this case the splines are monotonically increasing and constrained such that $c(0)=1$ and $c(1)=1$, while the free parameters are composed of the $y$ values for two fixed values of $x$, and the derivatives at the points $x=0$ and $x=1$. These four optimization parameters are schematized in Figure 3(a) and called $y_{1}=c\left(x_{1}\right), y_{2}=c\left(x_{2}\right), \sigma$ and $\rho$, respectively. As the splines are intended to be monotonically increasing, parameter $y_{2}$ is restricted to be equal to or greater than $y_{1}$. Then, parameter $y_{2}$ is obtained as $y_{2}=y_{1}+\delta_{y_{2}}$, and the parameters which are coded in the chromosomes are $y_{1}, \delta_{y_{2}}, \sigma$, and $\rho$. Given a particular chromosome, which sets the values of these parameters, the $y[i]$ corresponding to the $x[i]$ for all $i=1, \ldots, n_{f}$ are obtained by spline interpolation, using [25]

$$
y[i]=P[i] y_{1}+Q[i] y_{2}+R[i] y_{1}^{\prime \prime}+S[i] y_{2}^{\prime \prime},
$$

where $y_{1}^{\prime \prime}$ and $y_{2}^{\prime \prime}$ are the second derivatives at points $y_{1}$ and $y_{2}$, respectively. $P[i], Q[i], R[i]$, and $S[i]$ are defined by

$$
\begin{aligned}
& P[i] \triangleq \frac{x_{2}-x[i]}{x_{2}-x_{1}}, \quad R[i] \triangleq \frac{1}{6}\left((P[i])^{3}-P[i]\right)\left(x_{2}-x_{1}\right)^{2}, \\
& Q[i] \triangleq 1-P[i], \quad S[i] \triangleq \frac{1}{6}\left((Q[i])^{3}-Q[i]\right)\left(x_{2}-x_{1}\right)^{2} .
\end{aligned}
$$



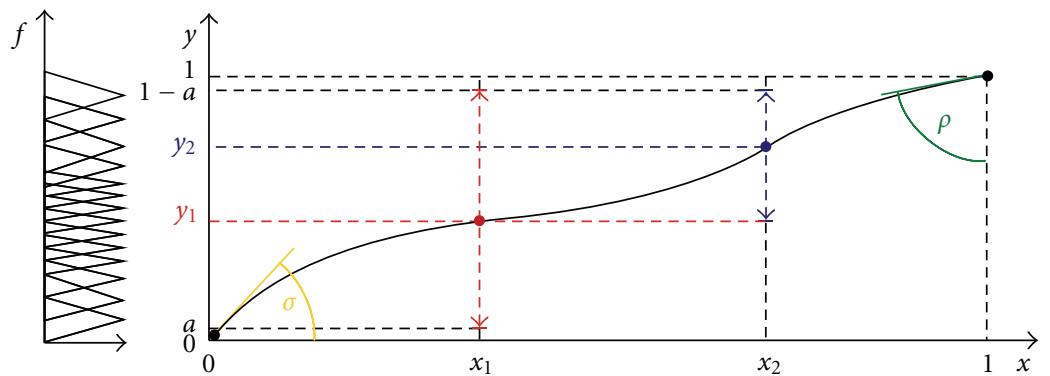

(a)

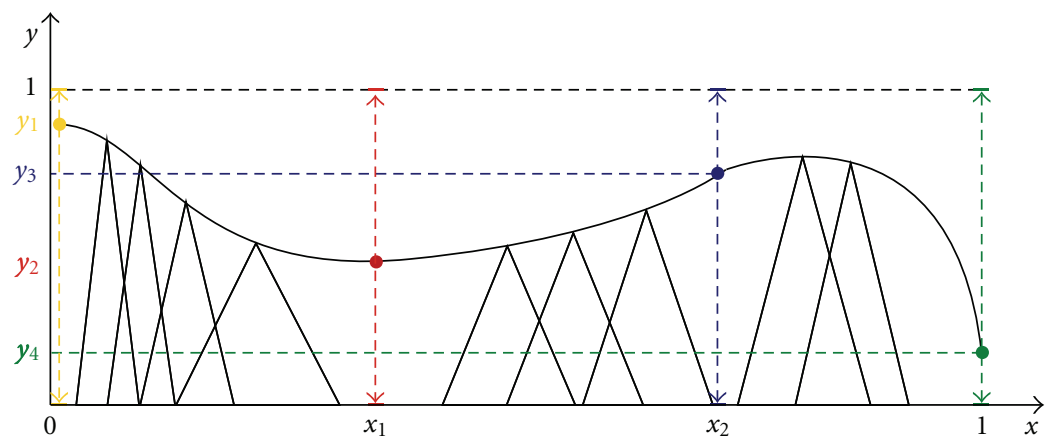

(b)

FIGURE 3: Schemes illustrating the use of splines to optimize the filterbanks. (a) A spline being optimized to determine the frequency position of filters, and (b) a spline being optimized to determine the amplitude of the filters.

However, the second derivatives $y_{1}^{\prime \prime}$ and $y_{2}^{\prime \prime}$, which are generally unknown, are required in order to obtain the interpolated values $y[i]$ using (3). In the case of cubic splines the first derivative is required to be continuous across the boundary of two intervals, and this requirement allows to obtain the equations for the second derivatives [25]. The required equations are obtained by setting the first derivative of (3) evaluated for $x_{j}$ in the interval $\left(x_{j-1}, x_{j}\right)$ equal to the same derivative evaluated for $x_{j}$ but in the interval $\left(x_{j}, x_{j+1}\right)$. This way a set of linear equations is obtained, for which it is necesary to set boundary conditions for $x=0$ and $x=1$ in order to obtain a unique solution. These boundary conditions may be set by fixing the $y$ values for $x=0$ and $x=1$, or the values for the derivative $\sigma$ and $\rho$.

All the $y[i]$ are then linearly mapped to the frequency range of interest, namely, from $0 \mathrm{~Hz}$ to half sampling frequency $\left(f_{s}\right)$, in order to adjust the frequency values where the $n_{f}$ filters reach their maximum, $f_{i}^{c}$ :

$$
f_{i}^{c}=\frac{\left(y[i]-y_{\min }\right) f_{s}}{y_{\max }-y_{\min }},
$$

where $y_{\min }$ and $y_{\max }$ are the spline minimum and maximum values, respectively. As can be seen in Figure 3(a), for segments where $y$ increases fast the filters are far from each other, and for segments where $y$ increases slowly the filters are closer together. Parameter $a$ in Figure 3(a) controls the range of $y_{1}$ and $y_{2}$ (and $\delta_{y_{2}}$ ), and it is set in order to reduce the number of splines with $y$ values outside of $[0,1]$. The chromosomes which produce splines that go beyond the boundaries are penalized, and the corresponding curves are

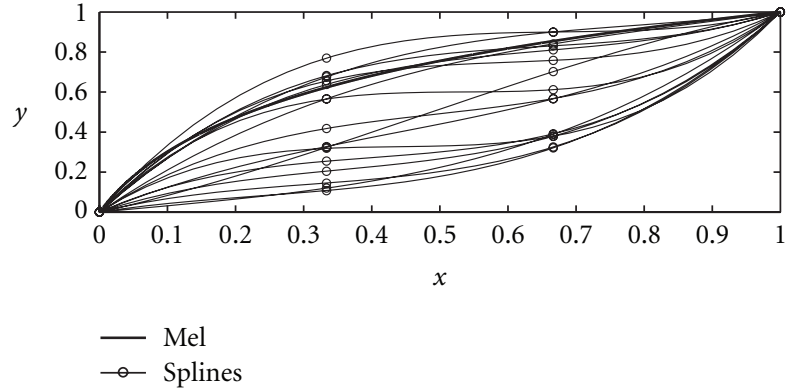

FIgUre 4: Mel-scale and spline-scale examples comparison.

modified so that $y$ values lower than 0 are set to 0 while values greater than 1 are set to 1 . Figure 4 shows some examples of splines that meet the restrictions and they are compared with the classical mel mapping. Note that on the $x$-axis, $n_{f}$ equidistant points are considered, and the $y$-axis is mapped to frequency in hertz, from zero to the Nyquist frequency.

Splines for Optimizing the Amplitude of the Filters. The only restriction for these splines is that $y$ varies in the range $[0,1]$, and the values at $x=0$ and $x=1$ are not fixed. So, in this case the optimization parameters are the four corresponding values $y_{1}, y_{2}, y_{3}$, and $y_{4}$ for the fixed values $x_{1}, x_{2}, x_{3}$, and $x_{4}$. These four $y_{j}$ parameters vary in the range $[0,1]$. Here, the interpolated $y[i]$ values directly determine the gain of each of the $n_{f}$ filters. This is outlined in Figure 3(b), where the gain of each filter is weighted according to the spline. 
Thus, it is expected to enhance the frequency bands which are relevant for classification, while disregarding those that are noise-corrupted.

Note that, as will be explained in Section 3.2, using this codification the chromosome size is reduced from $n_{f}$ to 4 . For instance, for a typical number of filters the chromosome size is reduced from 30 to 4 . Moreover, for the complete scheme in which both filter positions and amplitudes are optimized, the chromosome size is reduced from 60 to 8 genes. Indeed, with the splines codification the chromosome size is independent of the number of filters.

3.1. Adaptive Training and Test Subset Selection. In order to avoid the problem of overfitting during the optimization, we incorporate an adaptation of the training subset selection method similar to the one proposed in [26]. The filterbank parameters are evolved on selected subsets of training and test patterns, which are modified throughout the optimization. In every EA generation, training and test subsets are randomly selected for the fitness calculation, giving more chance to the test cases that were previously misclassified and to those that have not been selected for several generations. This strategy enables us to evolve filterbanks with more variety, giving generalization without increasing computational cost.

This is implemented by assigning a probability to each training/test case. In the first generation, the probabilities are initialized to the same value for all cases. For the training set, the probabilities are fixed during the optimization, while the probabilities for the test cases are updated every generation. In this case, for generation $g$ the probability of selection for test case $k$ is given by

$$
P_{k}(g)=\frac{W_{k}(g) S}{\sum_{j} W_{j}(g)},
$$

where $W_{k}(g)$ is the weight assigned to test case $k$ in generation $g$, and $S$ is the size of the subset selected. The weight for a test case $k$ is obtained by

$$
W_{k}(g)=D_{k}(g)+A_{k}(g),
$$

where $D_{k}(g)$ (difficulty of test case $k$ ) counts the number of times that test case $k$ misclassified, and $A_{k}(g)$ (age of test case $k$ ) counts the number generations since test case $k$ was selected for the last time. For every generation, the age of every unselected case is incremented by 1 , and the age of every selected case is set to 1 .

3.2. Description of the Optimization Process. In the EA population, every individual encodes the parameters of the splines that represent the different filterbanks, giving a particular formula for the ESCC. A chromosome is coded as a string of real numbers, its size is given by the number of optimized splines multiplied by the number of spline parameters, and they are initialized by means of a random uniform distribution. In the following section we show optimized filterbanks obtained by means of one and two splines. In the case of one spline we optimized only the

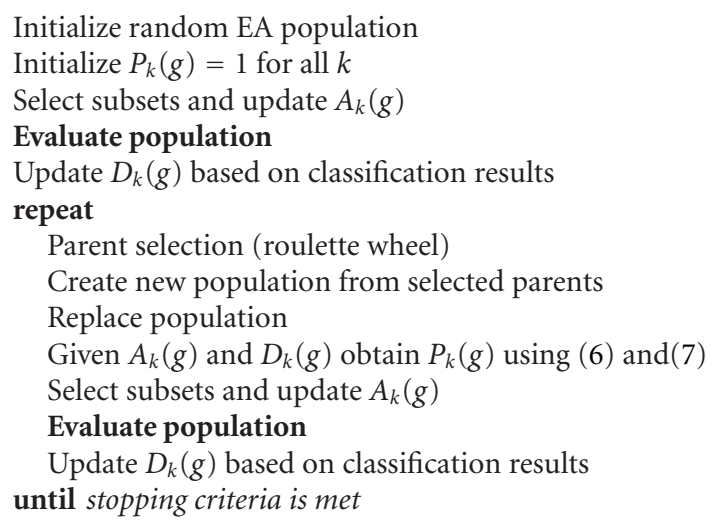

Algorithm 1: Optimization for ESCC.

frequency position of the filters and in the case of two splines we optimized both the frequency position and the filter amplitudes. For these cases, the chromosomes were of size 4 and 8 , respectively.

The EA uses the roulette wheel selection method [27], and elitism is incorporated into the search due to its proven capabilities to enforce the algorithm convergence under certain conditions [18]. The elitist strategy consists in maintaining the best individual from one generation to the next. The variation operators used in this EA are mutation and crossover, and they were implemented as follows. Mutation consists in the random modification of a random spline parameter, using a uniform distribution. The classical one-point crossover operator interchanges spline parameters between different chromosomes. The selection process should assign greater probability to the chromosomes providing the best filterbanks, and these will be the ones that facilitate the classification task. The fitness function consists of a phoneme classifier, and the fitness value of an individual is its success rate.

The steps for the filterbank optimization are summarized in Algorithm 1, and the details for the population evaluation are shown in Algorithm 2.

\section{Results and Discussion}

Many different experiments were carried out in order to find an optimal filterbank for the task of phoneme recognition. In this section we discuss the EA runs which produced the most interesting results and compare the obtained ESCC to the classic MFCC on the same classification tasks.

4.1. Speech Data. Phonetic data was extracted from the TIMIT speech database [28] and selected randomly from all dialect regions, including both male and female speakers. Utterances were phonetically segmented to obtain individual files with the temporal signal of every phoneme occurrence. White noise was also added at different SNR levels. The sampling frequency was $16 \mathrm{kHz}$ and the frames were extracted using a Hamming window of 25 milliseconds (400 samples) 


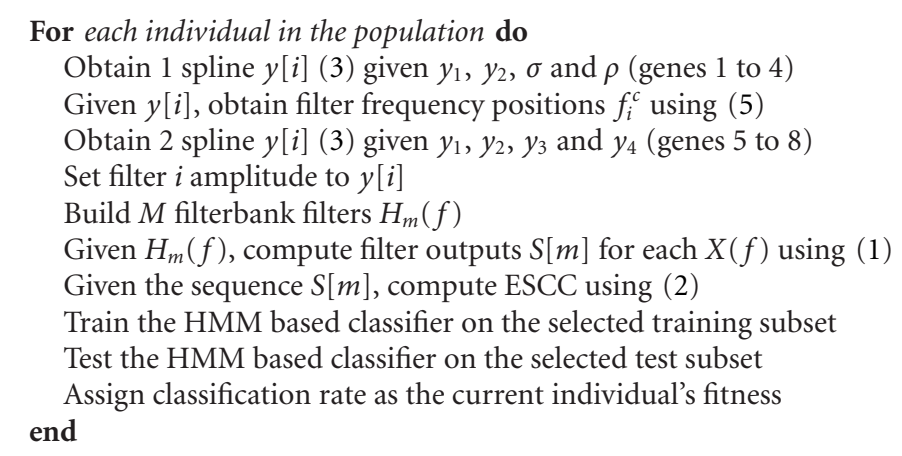

Algorithm 2: Evaluate population.

and a step-size of 200 samples. All possible frames within a phoneme occurrence were extracted and padded with zeros where necessary. The set of English phonemes /b/, /d/, /eh/, /ih/, and /jh/ was considered. Occlusive consonants /b/ and /d/ were included because they are very difficult to distinguish in different contexts. Phoneme /jh/ presents special features of the fricative sounds. Vowels /eh/ and /ih/ are commonly chosen because they are close in the formant space. As a consequence, this phoneme set consists of a group of classes which is difficult for automatic recognition [29].

4.2. Experimental Setup. Our phoneme classifier is based on continuous HMM, using Gaussian mixtures with diagonal covariance matrices for the observation densities [30]. For the experiments, we used a three-state HMM and mixtures of four gaussians. This fitness function uses tools from the HMM Toolkit (HTK) [31] for building and manipulating hidden Markov models. These tools implement the BaumWelch algorithm [32] which is used to train the HMM parameters, and the Viterbi algorithm [33] which is used to search for the most likely state sequence, given the observed events, in the recognition process.

In all the EA runs the population size was set to 30 individuals, crossover rate was set to 0.9 , and the mutation rate was set to 0.07 . Parameter $a$, discussed in the previous section, was set to 0.1 . For the optimization, a changing set of 1000 signals (phoneme examples) was used for training and a changing set of 400 signals was used for testing. Both sets were class-balanced and resampled every generation. The resampling of the training set was made randomly from a set of 5000 signals, and the resampling of the testing set was made taking into account previous misclassifications and the age of each of 1500 signals. The age of a signal was defined as the number of generations since it was included in the test set. The termination criterion for an EA run was to stop the optimization after 2500 generations. At termination, the filterbanks with the best fitness values were chosen.

Further cross-validation tests with ten different data partitions, consisting of 2500 training signals and 500 test signals each, were conducted with selected filterbanks. Two different validation tests were employed: match training
(MT), where the SNR was the same in both training and test sets, and mismatch training (MMT), which means testing with noisy signals (at different SNR levels) using a classifier that was trained with clean signals. From these validation tests we selected the best filterbanks, discarding those that were overoptimized (i.e., those with higher fitness but with lower validation result). Averaged validation results for the best optimized filterbanks were compared with the results achieved with the standard MFB on the same ten data partitions and training conditions. Note that, in all these experiments, the classifier was evaluated in MT conditions during the evolution.

4.3. Optimization of Central Frequencies. In the first experiment only the frequency positions of the filters were optimized, with chromosomes of length 4 (as explained in the previous section). The gain of each filter was not optimized; so, as in the case of the MFCC, every filter amplitude was scaled according to its bandwidth. Note that the number of filters in the filterbanks is not related to the size of the chromosomes. We considered filterbanks composed of 30 filters, while the feature vectors consisted of the first 16 cepstral coefficients. In this case, clean signals were used to train and test the classifier during the optimization.

Table 1 summarizes the validation results for evolved filterbanks (EFB) EFB-A1, EFB-A2, EFB-A3, and EFBA4, which are the best from the first experiment. Their performance is compared with that of the classic filterbank on different noise and training conditions. As can be seen, in most test cases the optimized filterbanks perform better than MFB, specially for match training tests. Figure 5 shows these four EFBs, which exhibit little difference between them. Moreover, their frequency distributions are similar to that of the classical MFB. However, the resolution that these filterbanks provide below $2 \mathrm{kHz}$ is higher, probably because this is the place for the two first formant frequencies. In contrast, when polynomial functions were used to encode the parameters [13], the obtained filterbanks were not regular and did not always cover most of the frequency band of interest. This may be attributed to the complex relation between filterbank parameters and the optimized polynomials. 
TABLE 1: Averaged validation results for phoneme recognition (shown in percent). Filterbanks are obtained from the optimization of filter center frequency values, while filter gains-scaled according to bandwidths and using clean signals.

\begin{tabular}{lccccccccccc}
\hline \multirow{2}{*}{ FB } & \multirow{3}{*}{$n_{f}$} & $n_{c}$ & \multicolumn{4}{c}{ Match training validation } & \multicolumn{4}{c}{ Mismatch training validation } \\
& & & $0 \mathrm{~dB}$ & $10 \mathrm{~dB}$ & $20 \mathrm{~dB}$ & $30 \mathrm{~dB}$ & clean & $0 \mathrm{~dB}$ & $10 \mathrm{~dB}$ & $20 \mathrm{~dB}$ & $30 \mathrm{~dB}$ \\
\hline EFB-A1 & 30 & 16 & 73.14 & $\mathbf{7 8 . 0 6}$ & $\mathbf{7 3 . 5 4}$ & $\mathbf{7 0 . 7 4}$ & $\mathbf{7 0 . 9 4}$ & $\mathbf{2 3 . 8 6}$ & 44.06 & $\mathbf{6 9 . 6 6}$ & $\mathbf{7 0 . 5 4}$ \\
EFB-A2 & 30 & 16 & 73.36 & 77.94 & $\mathbf{7 3 . 5 2}$ & $\mathbf{7 1 . 6 0}$ & $\mathbf{7 1 . 1 6}$ & 22.98 & 43.14 & $\mathbf{7 0 . 5 2}$ & $\mathbf{7 1 . 4 0}$ \\
EFB-A3 & 30 & 16 & $\mathbf{7 3 . 6 0}$ & $\mathbf{7 8 . 0 8}$ & $\mathbf{7 3 . 3 6}$ & $\mathbf{7 1 . 1 4}$ & $\mathbf{7 1 . 0 0}$ & 23.62 & 44.14 & $\mathbf{6 9 . 9 4}$ & $\mathbf{7 1 . 2 8}$ \\
EFB-A4 & 30 & 16 & 72.88 & $\mathbf{7 8 . 0 4}$ & $\mathbf{7 3 . 5 6}$ & $\mathbf{7 1 . 4 6}$ & $\mathbf{7 1 . 9 2}$ & 23.68 & 43.80 & $\mathbf{7 0 . 0 6}$ & $\mathbf{7 1 . 2 8}$ \\
MFB & 30 & 16 & 73.44 & 77.88 & 71.22 & 70.20 & 69.94 & 23.72 & $\mathbf{4 4 . 7 4}$ & 66.60 & 70.38 \\
\hline
\end{tabular}

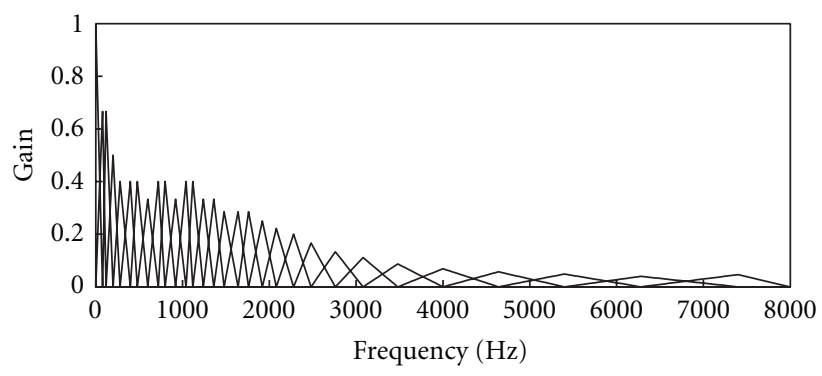

(a)

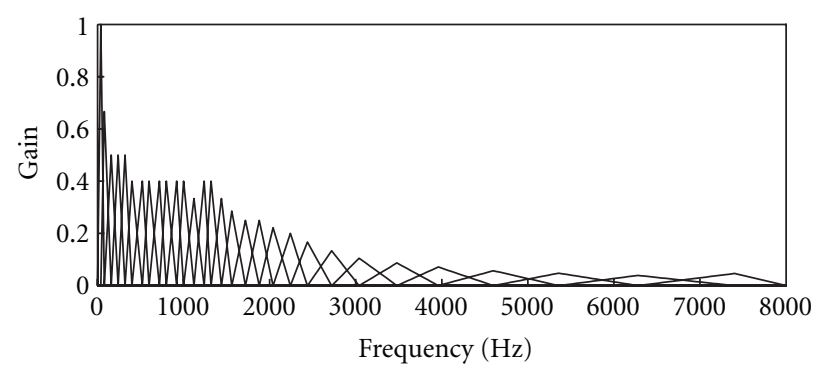

(c)

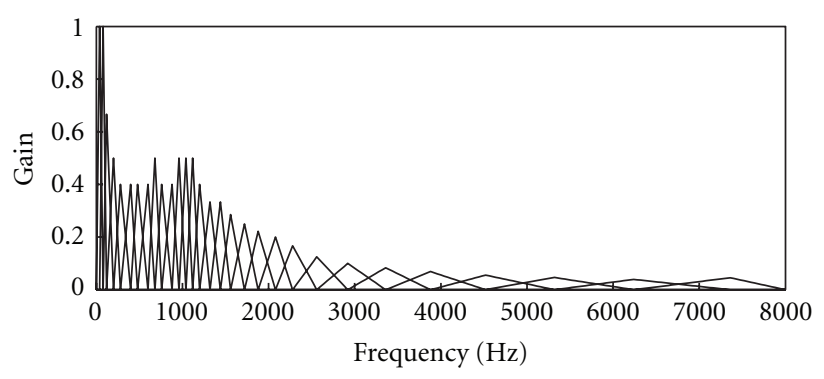

(b)

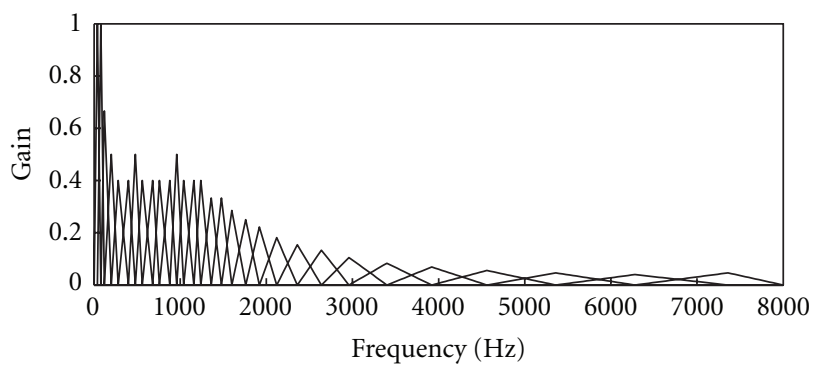

(d)

FIGURE 5: Evolved filterbanks obtained in the optimization of filter center positions only (filter gains normalized according to bandwidths) using clean signals. (a) EFB-A1, (b) EFB-A2, (c) EFB-A3 and (d) EFB-A4.

4.4. Optimization of Filter Gain and Center Frequency. The second experiment differs only in that the filters' amplitude was also optimized, coding the parameters of two splines in each chromosome of length 8. Validation results for EFBB1, EFB-B2, EFB-B3, and EFB-B4 are shown in Table 2, from which important improvements over the classical filterbank can be appreciated. Each of the optimized filterbanks performs better than MFB in most of the test conditions. For the MT cases of $20 \mathrm{~dB}, 30 \mathrm{~dB}$, and clean, and for the MMT case of $10 \mathrm{~dB}$ the improvements are most significant. These four EFBs, which can be observed in Figure 6, differ from MFB (shown in Figure 2) in the scaling of the filters at higher frequencies. Moreover, these filterbanks emphasize the highfrequency components. As in the case of those in Figure 5, these EFBs show more filter density before $2 \mathrm{kHz}$, compared to MFB.

In the third experiment both the frequency positions and amplitude of the filters were optimized (as in the previous case). However, in this case noisy signals at $0 \mathrm{~dB}$ SNR were used to train and test the classifier during the evolution.
Validation results from Table 3 reveal that for the case of $0 \mathrm{~dB}$ SNR, in both MT and MMT conditions, these EFBs improve the ones in Tables 1 and 2. The filterbanks optimized on clean signals perform better for most of the noise contaminated conditions.

These EFBs are more regular compared to those obtained in previous works, where the optimization considered three parameters for each filter [17]. These parameters were the frequency positions at the initial, top, and end points of the triangular filters, while size and overlap were left unrestricted. Results showed some phoneme classification improvements, although the shapes of optimized filterbanks were not easy to explain. Moreover, dissimilar filterbanks gave comparable results, showing that we were dealing with an ill-conditioned problem. This was particularly true when the optimization was made using noisy signals, as the solution does not continuously depend on data. In this work, dissimilarities between EFBs are only noticeable for those filterbanks that were optimized using noisy signals. 
TABLE 2: Averaged validation results for phoneme recognition (shown in percent). Filterbanks are obtained from the optimization of filter center frequency and filter gain values and using clean signals.

\begin{tabular}{lcccccccccccc}
\hline \multirow{2}{*}{ FB } & \multirow{2}{*}{$n_{f}$} & $n_{c}$ & \multicolumn{4}{c}{ Match training validation } & \multicolumn{4}{c}{ Mismatch training validation } \\
& & & $0 \mathrm{~dB}$ & $10 \mathrm{~dB}$ & $20 \mathrm{~dB}$ & $30 \mathrm{~dB}$ & clean & & $0 \mathrm{~dB}$ & $10 \mathrm{~dB}$ & $20 \mathrm{~dB}$ & $30 \mathrm{~dB}$ \\
\hline EFB-B1 & 30 & 16 & 73.06 & $\mathbf{7 8 . 4 0}$ & $\mathbf{7 8 . 5 6}$ & $\mathbf{7 5 . 5 2}$ & $\mathbf{7 4 . 1 6}$ & 22.94 & $\mathbf{4 5 . 7 0}$ & 55.44 & $\mathbf{7 1 . 8 0}$ \\
EFB-B2 & 30 & 16 & $\mathbf{7 3 . 7 6}$ & $\mathbf{7 8 . 3 8}$ & $\mathbf{7 9 . 0 8}$ & $\mathbf{7 6 . 2 6}$ & $\mathbf{7 4 . 8 4}$ & $\mathbf{2 4 . 2 6}$ & $\mathbf{5 0 . 1 6}$ & $\mathbf{6 4 . 8 4}$ & $\mathbf{7 3 . 1 0}$ \\
EFB-B3 & 30 & 16 & $\mathbf{7 3 . 5 4}$ & 77.60 & $\mathbf{7 8 . 0 4}$ & $\mathbf{7 6 . 0 2}$ & $\mathbf{7 4 . 2 8}$ & 22.56 & $\mathbf{4 7 . 3 2}$ & $\mathbf{6 3 . 8 2}$ & $\mathbf{7 0 . 6 0}$ \\
EFB-B4 & 30 & 16 & $\mathbf{7 3 . 7 4}$ & $\mathbf{7 8 . 7 4}$ & $\mathbf{7 9 . 1 8}$ & $\mathbf{7 5 . 6 6}$ & $\mathbf{7 5 . 4 0}$ & 23.22 & $\mathbf{5 1 . 4 6}$ & $\mathbf{6 6 . 5 8}$ & $\mathbf{7 2 . 9 6}$ \\
MFB & 30 & 16 & 73.44 & 77.88 & 71.22 & 70.20 & 69.94 & & 23.72 & 44.74 & $\mathbf{6 6 . 6 0}$ & 70.38 \\
\hline
\end{tabular}

TABLE 3: Averaged validation results for phoneme recognition (shown in percent). Filterbanks obtained from the optimization of filter center frequency and filter gain values, and using noisy signals.

\begin{tabular}{lcccccccccccc}
\hline \multirow{2}{*}{ FB } & \multirow{2}{*}{$n_{f}$} & $n_{c}$ & \multicolumn{4}{c}{ Match training validation } & \multicolumn{4}{c}{ Mismatch training validation } \\
& & & $0 \mathrm{~dB}$ & $10 \mathrm{~dB}$ & $20 \mathrm{~dB}$ & $30 \mathrm{~dB}$ & clean & & $0 \mathrm{~dB}$ & $10 \mathrm{~dB}$ & $20 \mathrm{~dB}$ & $30 \mathrm{~dB}$ \\
\hline EFB-C1 & 30 & 16 & $\mathbf{7 3 . 8 8}$ & 76.50 & $\mathbf{7 6 . 2 4}$ & $\mathbf{7 0 . 7 8}$ & 69.14 & $\mathbf{3 1 . 7 6}$ & 44.46 & 49.16 & 67.20 \\
EFB-C2 & 30 & 16 & $\mathbf{7 4 . 6 6}$ & $\mathbf{7 8 . 6 0}$ & $\mathbf{7 8 . 9 6}$ & $\mathbf{7 3 . 7 8}$ & $\mathbf{7 0 . 7 6}$ & $\mathbf{2 5 . 7 4}$ & $\mathbf{4 6 . 6 8}$ & 49.76 & 66.88 \\
EFB-C3 & 30 & 16 & $\mathbf{7 4 . 9 0}$ & 77.18 & $\mathbf{7 6 . 1 0}$ & $\mathbf{7 0 . 5 6}$ & 69.48 & $\mathbf{2 9 . 7 0}$ & 44.50 & 49.40 & 68.06 \\
EFB-C4 & 30 & 16 & $\mathbf{7 4 . 7 6}$ & $\mathbf{7 8 . 1 6}$ & $\mathbf{7 8 . 5 4}$ & $\mathbf{7 5 . 3 6}$ & $\mathbf{7 1 . 0 4}$ & $\mathbf{2 4 . 8 0}$ & $\mathbf{4 6 . 0 8}$ & 52.12 & $\mathbf{6 6 . 3 6}$ \\
MFB & 30 & 16 & 73.44 & 77.88 & 71.22 & 70.20 & 69.94 & & 23.72 & 44.74 & $\mathbf{6 6 . 6 0}$ & $\mathbf{7 0 . 3 8}$ \\
\hline
\end{tabular}

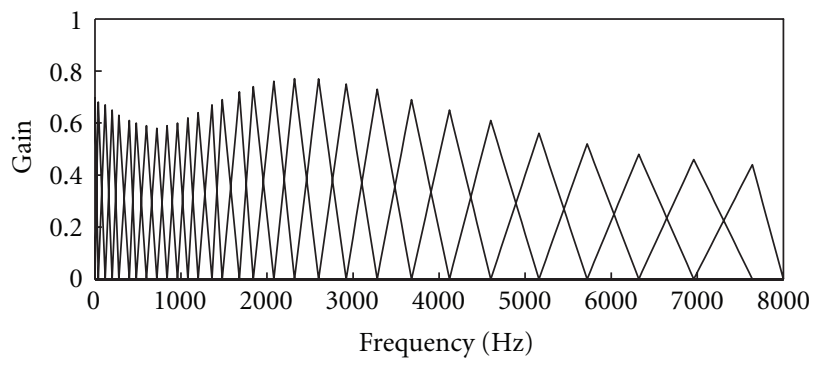

(a)

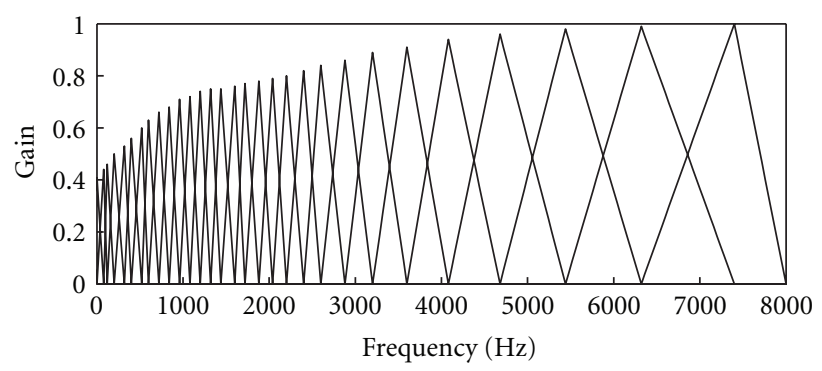

(c)

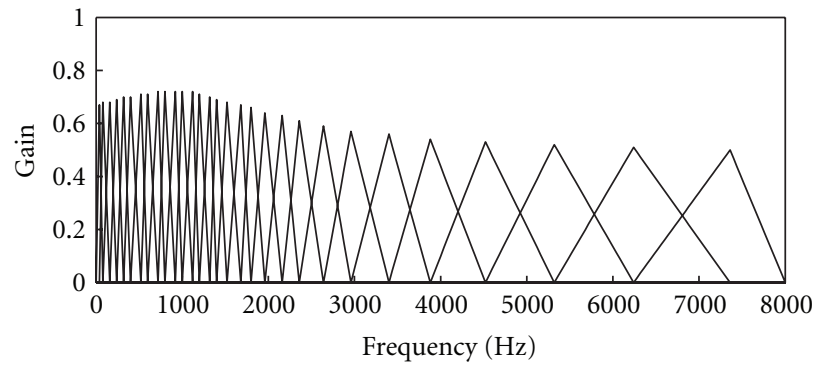

(b)

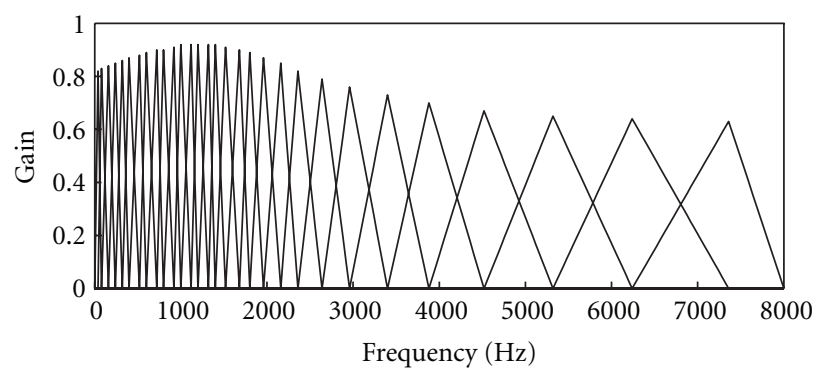

(d)

FIGURE 6: Evolved filterbanks obtained in the optimization of filter center positions and amplitudes simultaneously and using clean signals:

(a) EFB-B1, (b) EFB-B2, (c) EFB-B3, and (d) EFB-B4. 


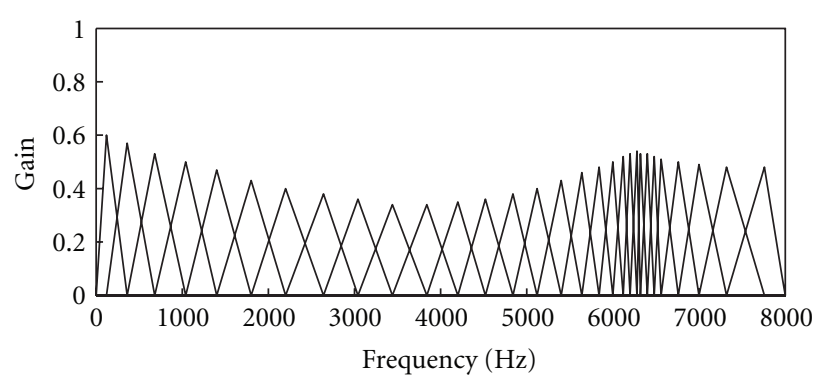

(a)

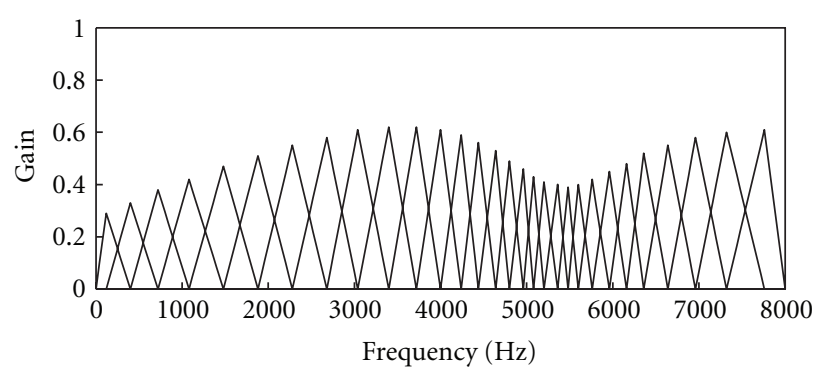

(c)

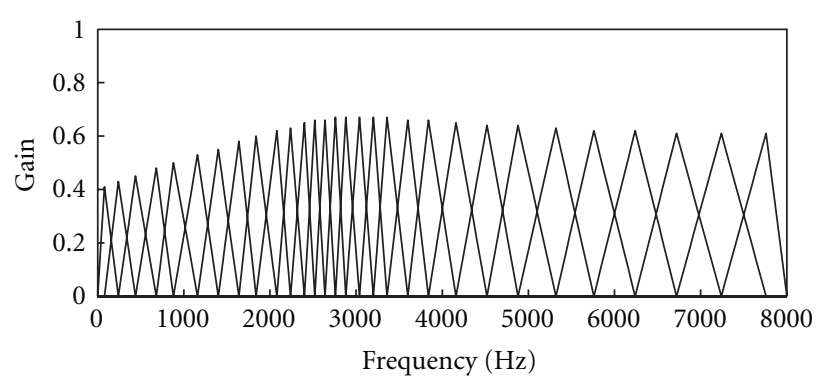

(b)

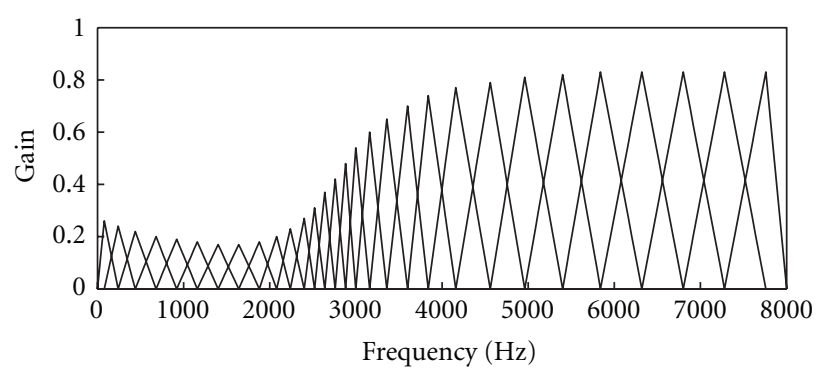

(d)

FIGURE 7: Evolved filterbanks obtained in the optimization of filter center positions and amplitudes simultaneously and using signals with noise at 0 dB SNR: (a) EFB-C1, (b) EFB-C2, (c) EFB-C3, and (d) EFB-C4.

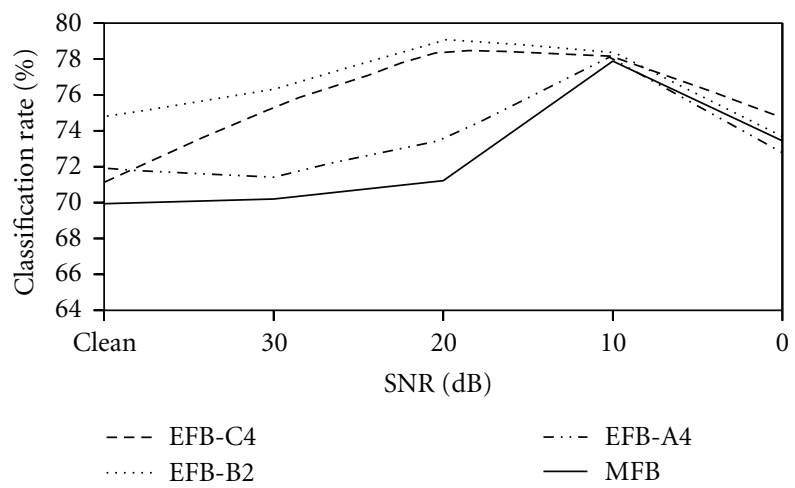

(a)

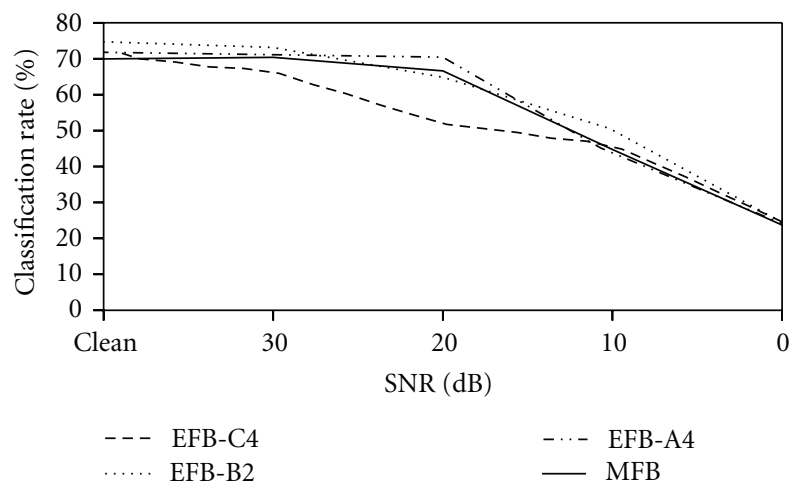

(b)

FIGURE 8: Averaged validation results for phoneme classification comparing MFB with EFB-A4, EFB-B2, and EFB-C4 at different training conditions. (a) Validation in match training conditions, and (b) validation in mismatch training conditions.

From Figure 7 we can observe that the filterbanks evolved on noisy signals differ widely from MFB and the ones evolved on clean signals. For example, the filter density is greater in different frequency ranges, and these ranges are centered in higher frequencies. Moreover, this amplitude scaling, in contrast to the preceding filterbanks, depreciates the lower-frequency bands. This feature is present in all these filterbanks, giving attention to high frequencies, as opposed to MFB, and taking higher formants into account. However, the noticeable dissimilarities in these four filterbanks suggest that the optimization with noisy signals is much more complex, preventing the EA to converge to similar solutions.
4.5. Analysis and Discussion. Figure 8 summarizes some results shown in Tables 1, 2, and 3 for EFB-A4, EFB-B2, and EFB-C4, and compares them with MFB on different noise and training conditions. From Figure 8(a) we can observe that, in MT conditions, the EFBs outperform MFB in almost all the noise conditions considered. Figure 8(b) shows some improvements of EFB-A4 and EFB-B2, over MFB, in MMT conditions.

Table 4 shows confusion matrices for phoneme classification with MFB and EFB-B2, from validation at various SNR levels in the MT case. From these matrices, one can notice that phonemes /b/, /eh/, and /ih/ are frequently misclassified 
TABLE 4: Confusion matrices showing percents of average classification rates from ten data partitions in MT conditions, for both MFB and EFB-B2.

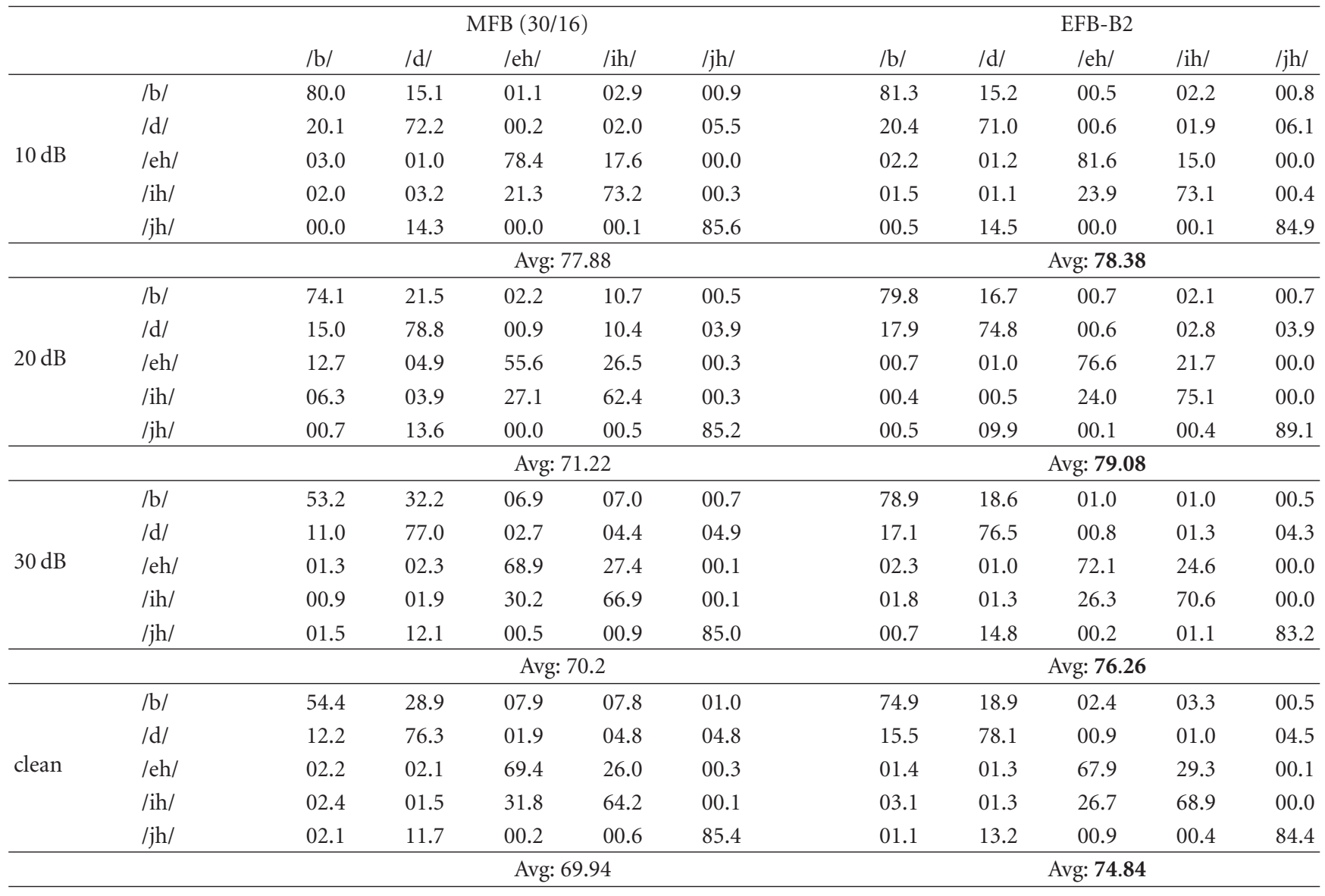

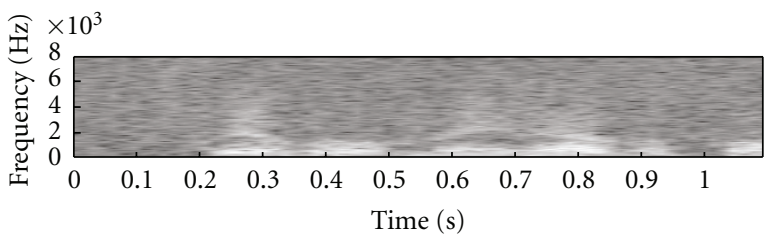

(a)

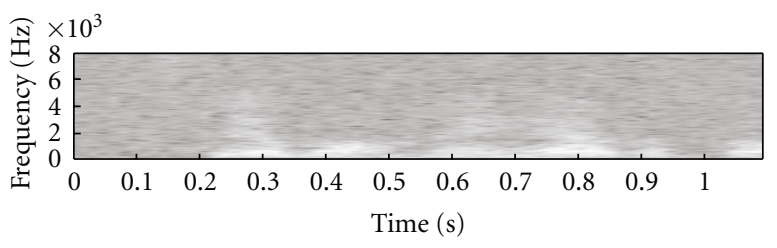

(b)

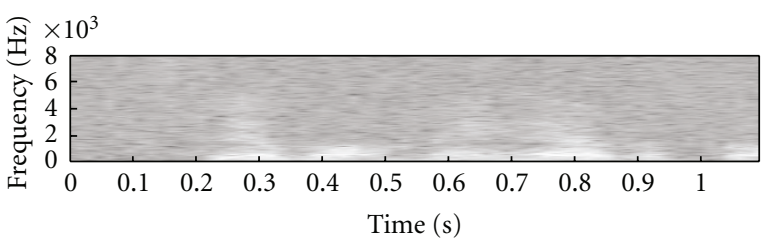

(c)

FIGURE 9: Spectrograms for a fragment of sentence SI648 from TIMIT corpus with additive white noise at $20 \mathrm{~dB}$ SNR. Computed from the original signal (a), reconstructed from MFCC (b) and reconstructed from EFB-B4 (c). using MFB and they are significantly better classified with EFB-B2. Moreover, with EFB-B2 the variance between the classification rates of individual phonemes is smaller. It can also be noticed that phoneme /b/ is mostly confused with phoneme /d/ and vice versa, and the same happens with vowels /eh/ and /ih/. This occurs with both filterbanks MFB and EFB-B4, though the optimized filterbank reduces these confusions considerably.

As these filterbanks were optimized for a reduced set of phonemes, one cannot a priori expect continuous speech recognition results to be improved. Thus, some preliminary tests were made and promising results were obtained. A recognition system was built using tools from HTK and the performance of the ESCC was compared to that of the classical MFCC representation, using sentences from dialect region one in TIMIT database with additive white noise at different SNRs (in MMT conditions). Preemphasis was applied to signal frames and the feature vectors were composed of the MFCC, or ESCC, plus delta and acceleration coefficients. The sentence and word recognition rates were close for MFCC and ESCC in almost all cases. At $15 \mathrm{~dB}$ the word recognition rates were $15.83 \%$ and $31.98 \%$ for MFB and EFB-B4, respectively. This suggests that even if the optimization is made over a small set of phonemes, the resulting feature set still allows us to better discriminate between other phoneme classes. Moreover, it is important 


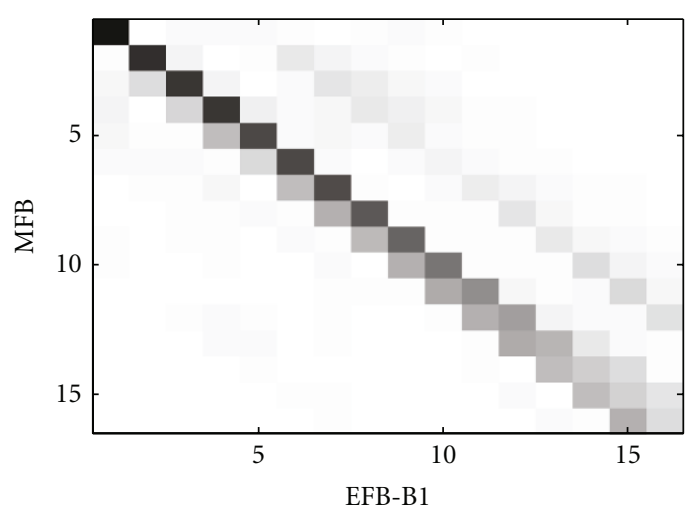

(a)

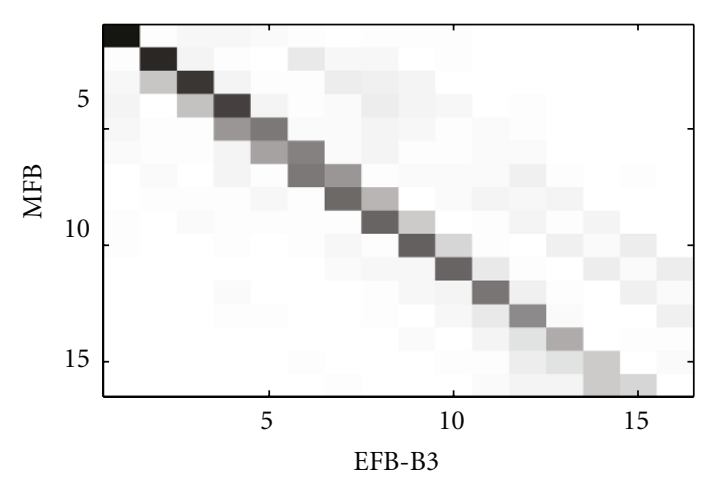

(c)

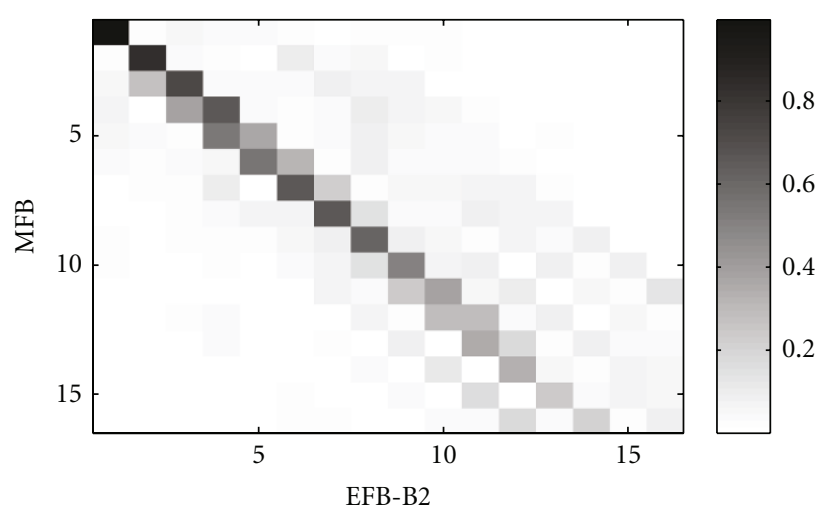

(b)

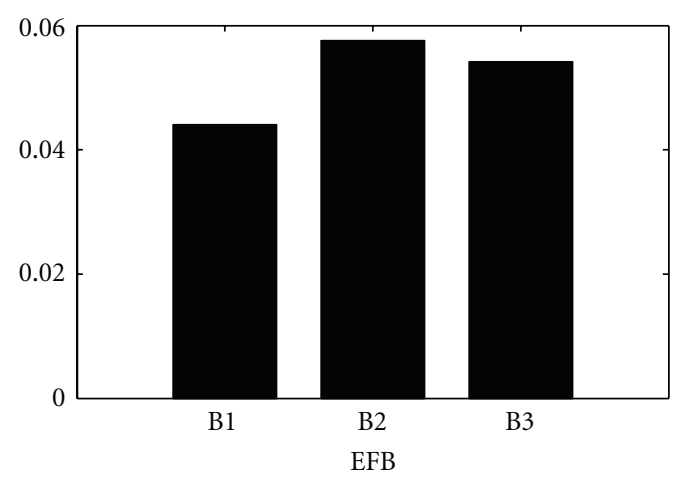

(d)

Figure 10: Squared Pearson's correlation between MFCC and ESCC obtained with EFB-B1, EFB-B2, and EFB-B3 ((a), (b) and (c), resp.). Normalized sum of the correlation coefficients outside the diagonal (d).

to note that the five phonemes selected for the filterbank optimization represent only $9.38 \%$ (b: $1.49 \%$, d: $2.28 \%$, eh: $2.35 \%$, ih: $2.76 \%$, jh: $0.51 \%$ ) of the total number of phonemes in the test utterances. That is, from a total of 3956 phonemes in the test utterances, only 371 correspond to the phoneme set considered in the optimization.

In order to understand the information that these filterbanks retain, an estimate of the short-time magnitude spectrum was recovered using the method proposed in [34]. This method scales the spectrogram of a white noise signal by the short-time magnitude spectrum recovered from the cepstral coefficients. The spectrograms for a fragment of sentence SI648 from TIMIT corpus with additive white noise at $20 \mathrm{~dB}$ SNR are shown in Figure 9. The spectrogram on top is the one corresponding to the original signal, in the middle the reconstructed spectrogram from MFCC is shown, and the one at the bottom was reconstructed from the ESCC obtained by means of EFB-B4. It can be observed that the spectrogram reconstructed from ESCC is less affected by noise than the other two. Moreover, the information from formant frequencies is enhanced and made easier to detect in the spectrogram corresponding to ESCC, which makes phoneme classification easier. This means that, in comparison to the MFB, the filter distribution and bandwidths of EFB-B4 allow more relevant information to be preserved.
In order to evaluate the relation of the MFCC and the ESCC we compared them using Pearson's correlation coefficient $r$. Figure 10 shows squared correlation matrices comparing the MFCC with the ESCC (obtained using EFBB1, EFB-B1, and EFB-B3) over 17846 phoneme frames with additive noise at $0 \mathrm{~dB}$ SNR. We observe that approximately the first half of the coefficients are quite highly correlated between the filterbanks under comparison. Moreover, in the case of EFB-B2 there are more correlation coefficients outside the diagonal which are different from zero. This means that the ESCC are obtained with EFB-B2 are the least related to the MFCC, in the sense that the information is distributed differently between all the cepstral coefficients. This can be better appreciated in the bar plot, giving the normalized sum of all the correlation coefficients outside the diagonal. Note that EFB-B2 is the one which gives the best validation results.

A similar comparison was made between the cepstral coefficients from a single filterbank, in order to evaluate how they are correlated. In Figure 11 the squared correlation matrices of the MFCC and the ESCC from EFB-B1, EFB-B2, and $\mathrm{EFB}-\mathrm{B} 3$ are shown. It can be noticed that the matrix for EFB-B2 is the one with the least number of coefficients different from zero outside the diagonal. Moreover, the normalized sum of the correlation coefficients outside the diagonal is smaller for EFB-B2, meaning that the ESCCs 


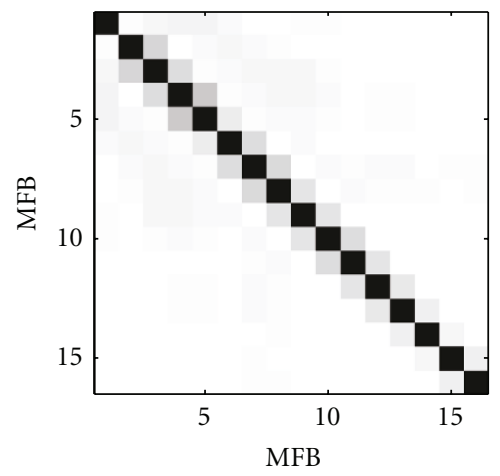

(a)

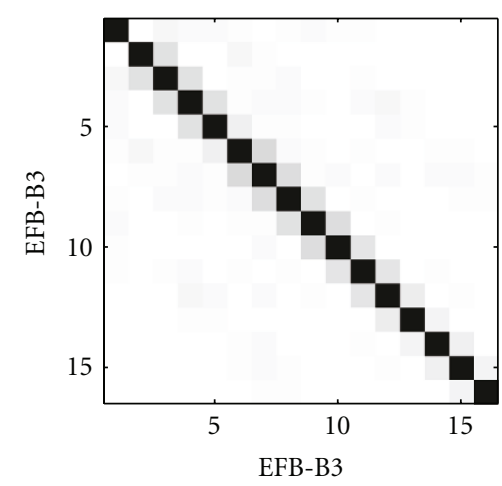

(d)

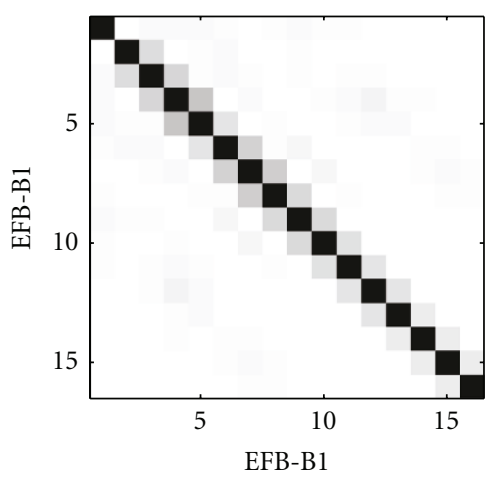

(b)

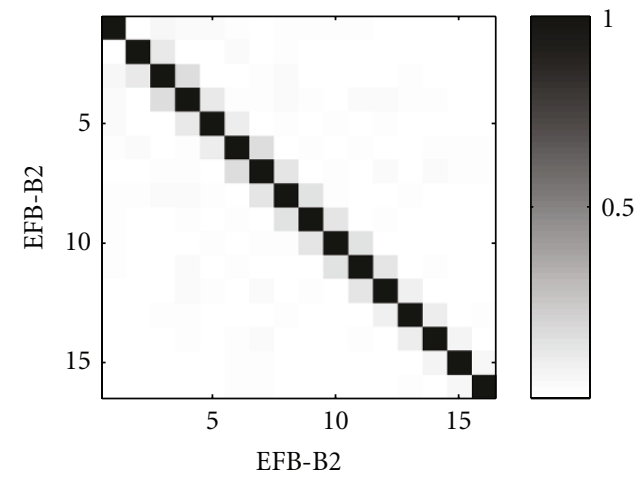

(c)

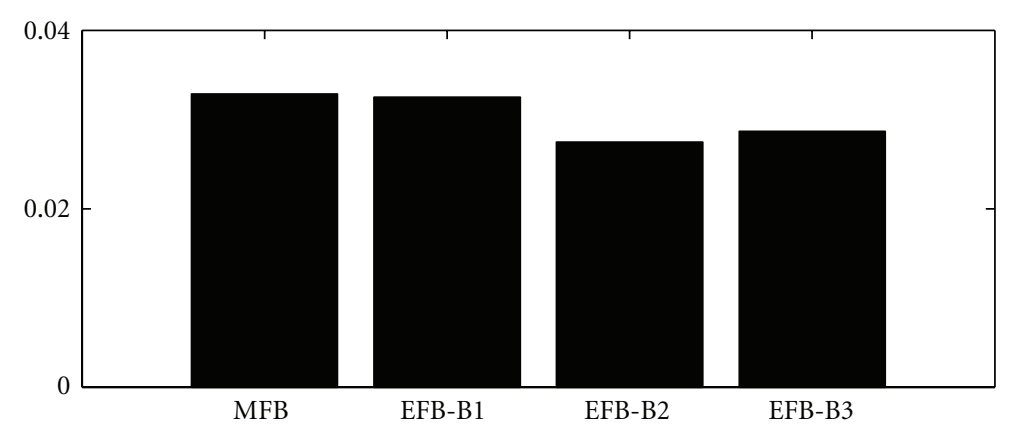

(e)

Figure 11: Squared Pearson's correlation of MFCC and ESCC obtained with EFB-B1, EFB-B2, and EFB-B3 ((a), (b), (c) and (d), resp.). Normalized sum of the correlation coefficients outside the diagonal (e).

from EFB-B2 are less correlated than MFCC. For this reason the ESCCs from EFB-B2 better satisfy the assumptions for HMM-based speech recognizers using GM observation densities with diagonal covariance matrices (a common practice in speech recognition) [30].

Another subject to consider is the computational load of the optimizations detailed in the previous section. An EA run of 2500 generations (which is the number of generations used in this work for the experiments) takes approximately 84 hours (about 2 minutes for each generation) on a computer cluster consisting of eleven processors of $3 \mathrm{GHz}$ clock speed. It is interesting to note that the most expensive computation in the optimization is the fitness evaluation, that is, the training and test of the HMM-based classifier. In comparison to the approach [17] (in which the filterbank parameters were directly coded in the chromosomes), the reduced chromosome size allowed the EA to converge to better solutions taking almost the same processing time. It is important to note that this approach does not imply additional load to the standard speech recognition procedure. The optimization step is previous to the recognition, and the filterbank is fixed during the entire recognition. Moreover, the MFCC and the ESCC feature extraction techniques are similar, and the optimization can be considered as part of the training.

\section{Conclusions}

In this work an evolutionary method has been proposed for the optimization of a filterbank, in order to obtain a new cepstral representation for phoneme classification. We introduced the use of a spline interpolation which reduces the number of parameters in the optimization, providing an adequate search space. The advantages of evolutionary computation are successfully exploited in the search for an optimal filterbank. The encoding of parameters by means of spline functions significantly reduced the chromosome size and search space, while preserving a broad variety of candidate solutions. Moreover, the suitable variation operators allowed the algorithm to explore a large pool of potential filterbanks.

Experimental results show that the proposed method is able to find a robust signal representation, which allows us to improve the classification rate for a given set of phonemes at different noise conditions. Furthermore, this strategy can provide alternative speech representations that improve the results of the classical approaches for specific conditions. These results also suggest that there is further room for improvement over the classical filterbank. On the other hand, with the use of these optimized filterbanks the robustness of an ASR system can be improved with no additional 
computational cost, and without modifications in the HMM structure or training algorithm.

Further work will include the utilization of other search methods, such as particle swarm optimization and scatter search [35]. In addition, different variation operators can be evaluated and other filter parameters such as bandwidth could also be optimized. The possibility of replacing the HMM-based classifier by another objective function of lower computational cost, such as a measure of class separability, will also be studied. Finally, future experiments will include the optimization using a bigger set of phonemes and further comparisons of the ESCC to classical features in the continuous speech recognition task.

\section{Acknowledgments}

The authors wish to thank their lab colleagues María Eugenia Torres and Leandro Di Persia for sharing their experience through their technical support and excellent advice, from which this work has benefited.

\section{References}

[1] S. B. Davis and P. Mermelstein, "Comparison of parametric representations for monosyllabic word recognition in continuously spoken sentences," IEEE Transactions on Acoustics, Speech, and Signal Processing, vol. 28, no. 4, pp. 357-366, 1980.

[2] F. Zheng, G. Zhang, and Z. Song, "Comparison of different implementations of MFCC," Journal of Computer Science and Technology, vol. 16, no. 6, pp. 582-589, 2001.

[3] M. D. Skowronski and J. G. Harris, "Exploiting independent filter bandwidth of human factor cepstral coefficients in automatic speech recognition," Journal of the Acoustical Society of America, vol. 116, no. 3, pp. 1774-1780, 2004.

[4] M. D. Skowronski and J. G. Harris, "Improving the filter bank of a classic speech feature extraction algorithm," in Proceedings of the IEEE International Symposium on Circuits and Systems (ISCAS '03), vol. 4, pp. 281-284, May 2003.

[5] H. Yeganeh, S. M. Ahadi, S. M. Mirrezaie, and A. Ziaei, "Weighting of mel sub-bands based on SNR/entropy for robust ASR," in Proceedings of the 8th IEEE International Symposium on Signal Processing and Information Technology (ISSPIT'08), pp. 292-296, December 2008.

[6] B. Nasersharif and A. Akbari, "SNR-dependent compression of enhanced Mel sub-band energies for compensation of noise effects on MFCC features," Pattern Recognition Letters, vol. 28, no. 11, pp. 1320-1326, 2007.

[7] XI. Zhou, Y. Fu, M. Liu, M. Hasegawa-Johnson, and T. S. Huang, "Robust analysis and weighting on MFCC components for speech recognition and speaker identification," in Proceedings of the IEEE International Conference onMultimedia and Expo (ICME '07), pp. 188-191, July 2007.

[8] H. Bořil, P. Fousek, and P. Pollák, "Data-driven design of front-end filter bank for Lombard speech recognition," in Proceedings of the 9th International Conference on Spoken Language Processing (ICSLP '06), pp. 381-384, Pittsburgh, Pa, USA, September 2006.

[9] Z. Wu and Z. Cao, "Improved MFCC-based feature for robust speaker identification,” Tsinghua Science \& Technology, vol. 10, no. 2, pp. 158-161, 2005.
[10] L. Burget and H. Heřmanský, "Data driven design of filter bank for speech recognition," in Text, Speech and Dialogue, vol. 2166 of Lecture Notes in Computer Science, pp. 299-304, Springer, Berlin, Germany, 2001.

[11] C. Charbuillet, B. Gas, M. Chetouani, and J. L. Zarader, "Optimizing feature complementarity by evolution strategy: application to automatic speaker verification," Speech Communication, vol. 51, no. 9, pp. 724-731, 2009.

[12] L. Vignolo, D. Milone, H. Rufiner, and E. Albornoz, "Parallel implementation for wavelet dictionary optimization applied to pattern recognition," in Proceedings of the 7th Argentine Symposium on Computing Technology, Mendoza, Argentina, 2006.

[13] C. Charbuillet, B. Gas, M. Chetouani, and J. L. Zarader, "Multi filter bank approach for speaker verification based on genetic algorithm," in Advances in Nonlinear Speech Processing, vol. 4885 of Lecture Notes in Computer Science, pp. 105-113, 2007.

[14] D. B. Fogel, Evolutionary Computation, John Wiley \& Sons, New York, NY, USA, 3rd edition, 2006.

[15] M. S. Lewicki, "Efficient coding of natural sounds," Nature Neuroscience, vol. 5, no. 4, pp. 356-363, 2002.

[16] L. D. Stein, "End of the beginning," Nature, vol. 431, no. 7011, pp. 915-916, 2004.

[17] L. D. Vignolo, H. L. Rufiner, D. H. Milone, and J. C. Goddard, "Genetic optimization of cepstrum filterbank for phoneme classification," in Proceedings of the 2nd International Conference on Bio-Inspired Systems and Signal Processing (BIOSIGNALS '09), pp. 179-185, Porto, Portugal, January 2009.

[18] T. Bäck, Evolutionary Algorithms in Theory and Practice: Evolution Strategies, Evolutionary Programming, Genetic Algorithms, Oxford University Press, Oxford, UK, 1996.

[19] T. Bäck, U. Hammel, and H. P. Schwefel, "Evolutionary computation: comments on the history and current state," IEEE Transactions on Evolutionary Computation, vol. 1, no. 1, pp. 3-17, 1997.

[20] Z. Michalewicz, Genetic Algorithms + Data Structures = Evolution Programs, Springer, Berlin, Germany, 1992.

[21] C. R. Jankowski Jr., H. D. H. Vo, and R. P. Lippmann, "A comparison of signal processing front ends for automatic word recognition," IEEE Transactions on Speech and Audio Processing, vol. 3, no. 4, pp. 286-293, 1995.

[22] L. Rabiner and B.-H. Juang, Fundamentals of Speech Recognition, Prentice Hall PTR, Englewood Cliffs, NJ, USA, 1993.

[23] J. R. Deller, J. G. Proakis, and J. H. Hansen, Discrete-Time Processing of Speech Signals, Macmillan, New York, NY, USA, 1993.

[24] M. Slaney, “Auditory Toolbox, version 2," Tech. Rep. 1998-010, Interval Research Corporation, Apple Computer Inc., 1998.

[25] W. H. Press, B. P. Flannery, S. A. Teukolsky, and W. T. Vetterling, Numerical Recipes in C: The Art of Scientific Computing, Cambridge University Press, Cambridge, UK, 2nd edition, 1992.

[26] C. Gathercole and P. Ross, "Dynamic training subset selection for supervised learning in genetic programming," in Parallel Problem Solving from Nature-PPSN III, vol. 866 of Lecture Notes in Computer Science, pp. 312-321, Springer, Berlin, Germany, 1994.

[27] A. E. Eiben and J. E. Smith, Introduction to Evolutionary Computing, Springer, Berlin, Germany, 2003.

[28] J. S. Garofalo, L. F. Lamel, W. M. Fisher, J. G. Fiscus, D. S. Pallett, and N. L. Dahlgren, "DARPA TIMIT acoustic phonetic continuous speech corpus CD-ROM,” Tech. Rep., U.S. Dept. of Commerce, NIST, Gaithersburg, Md, USA, 1993. 
[29] K. N. Stevens, Acoustic Phonetics, Mit Press, Cambrige, Mass, USA, 2000.

[30] K. Demuynck, J. Duchateau, D. van Compernolle, and P. Wambacq, "Improved feature decorrelation for HMM-based speech recognition," in Proceedings of the 5th International Conference on Spoken Language Processing (ICSLP '98), Sydney, Australia, 1998.

[31] S. Young, D. Kershaw, J. Odell, D. Ollason, V. Valtchev, and P. Woodland, HMM Toolkit, Cambridge University, 2000, http:// htk.eng.cam.ac.uk/.

[32] F. Jelinek, Statistical Methods for Speech Recognition, MIT Press, Cambrige, Mass, USA, 1999.

[33] X. D. Huang, Y. Ariki, and M. A. Jack, Hidden Markov Models for Speech Recognition, Edinburgh University Press, 1990.

[34] D. P. W. Ellis, "PLP and RASTA (and MFCC, and inversion) in Matlab,” http://www.ee.columbia.edu/ dpwe/resources/matlab/rastamat/.

[35] S. G. de los Cobos Silva, J. Goddard Close, M. A. Gutiérrez Andrade, and A. E. Martinez Licona, Búsqueda y Exploración Estocástica, Universidad Autónoma Metropolitana, Iztapalapa, Mexico, 1st edition, 2010. 